\title{
Technical improvements in the immunoperoxidase study of renal biopsy specimens
}

\author{
A J Howie, J Gregory, R A Thompson, M A Adkins, A J Niblett
}

\begin{abstract}
Sixty five renal biopsy specimens were used to compare a direct immunofluorescence technique on frozen sections with immunoperoxidase techniques on paraffin wax sections. For the immunoperoxidase techniques, dewaxed sections were treated with protease at $37^{\circ} \mathrm{C}$. Sections were examined at intervals on a microscope and digestion was stopped when plasma was removed from glomerular capillary loops. This permitted intense staining of immunoproteins on immunoperoxidase. There was agreement between immunoperoxidase and immunofluorescence in the staining for IgG, IgA, and IgM in 50 biopsy specimens and discordant findings did not affect the diagnosis. Immunoperoxidase did not detect $\mathrm{C3}$ in 16 biopsy specimens. Findings with an antiserum to another complement component, C9, detected by immunoperoxidase correlated with C3 findings detected by immunofluorescence in 17 biopsy specimens.

It is concluded that microscopical observation of the progress of digestion permits optimal staining by immunoperoxidase methods, thus overcoming the problem of variability in proteolytic digestion of sections. Inconsistency in the demonstration of complement deposition can be avoided by staining for $\mathrm{C} 9$ rather than C3.
\end{abstract}

Immunofluorescence on frozen sections is the oldest and most widely used technique for the immunological study of renal biopsy specimens. Immunoperoxidase techniques have advantages over immunofluorescence but are technically more difficult. ${ }^{1}$ Particular problems that we encountered were difficulties with optimal digestion of sections due to variable conditions of fixation of specimens and inconsistent demonstration of complement deposition.

M A Adkin

A J Niblett

Regional Department of Immunology, East Birmingham Hospital Birmingham

R A Thompson

Correspondence to:

Dr A J Howie, Department of Pathology, The Medical School, Birmingham B15 2TJ.

Accepted for publication 19 October 1989

\section{Methods}

To validate the immunoperoxidase method 65 renal biopsy specimens that had been initially studied by immunofluorescence were reexamined by immunoperoxidase. To compare detection of C3 and C9, 17 of these biopsy specimens and a further 17 were studied by immunoperoxidase.

\section{IMMUNOFLUORESCENCE}

Cryostat sections $6 \mu \mathrm{m}$ thick of each renal biopsy specimen frozen in liquid nitrogen were stained with a direct immunofluorescence technique $^{2}$ for $\operatorname{IgG}, \operatorname{IgA}, \operatorname{Ig} M$ and C3, using monospecific antisera conjugated with fluorescein isothiocyanate (Dako). Slides were examined on a Leitz fluorescence microscope with epi-illumination.

\section{IMMUNOPEROXIDASE}

Renal biopsy specimens were fixed in formolsaline, either by immersion at room temperature for at least 18 hours, or by immersion in boiling formol-saline shortly after the biopsy had been taken if an urgent diagnosis was essential for clinical reasons. Specimens were processed into paraffin wax on an Autotechnicon Ultra for rapid processing in two and a half hours or on a Shandon Hypercenter for orthodox processing overnight. Sections were examined by a three stage peroxidaseantiperoxidase (PAP) technique ${ }^{3}$ for IgG, IgA, and $\operatorname{IgM}$ and a two stage indirect immunoperoxidase technique ${ }^{3}$ for $\mathrm{C} 3$, after proteolytic digestion.

\section{PROTEOLYTIC DIGESTION}

Sections were cut at $2 \mu \mathrm{m}$ and dried on to glass slides at $60^{\circ} \mathrm{C}$ for at least 30 minutes. Sections were dewaxed in xylene, rehydrated through alcohols to water, and put in prewarmed $0.01 \mathrm{M}$ phosphate buffered saline, $\mathrm{pH} 7.2$ (PBS), at $37^{\circ} \mathrm{C}$ for five minutes. A prewarmed solution of protease type 24 (Sigma P8038), $0.5^{\circ}$ o in $\mathrm{PBS}$, was put on each slide at $37^{\circ} \mathrm{C}$ in an incubating chamber.

After three minutes, inspection of the progress of digestion was begun using either an orthodox microscope with the condenser adjusted to give maximum contrast or phasecontrast microscopy. A slide was removed from the chamber, rinsed with PBS, and examined microscopically. The first indication of digestion was granularity appearing in tubules. As digestion proceeded the glomerular basement membrane stood out as plasma was removed, giving a sharp contrast with the almost uniform, hyaline appearance of an undigested glomerulus (figs 1 and 2). Sometimes, red blood cells were also removed from capillary loops, enhancing the contrast.

If digestion was incomplete the section was replaced in the chamber and fresh protease was added. After a minute or so, depending on the rate of digestion, a different section of the same 


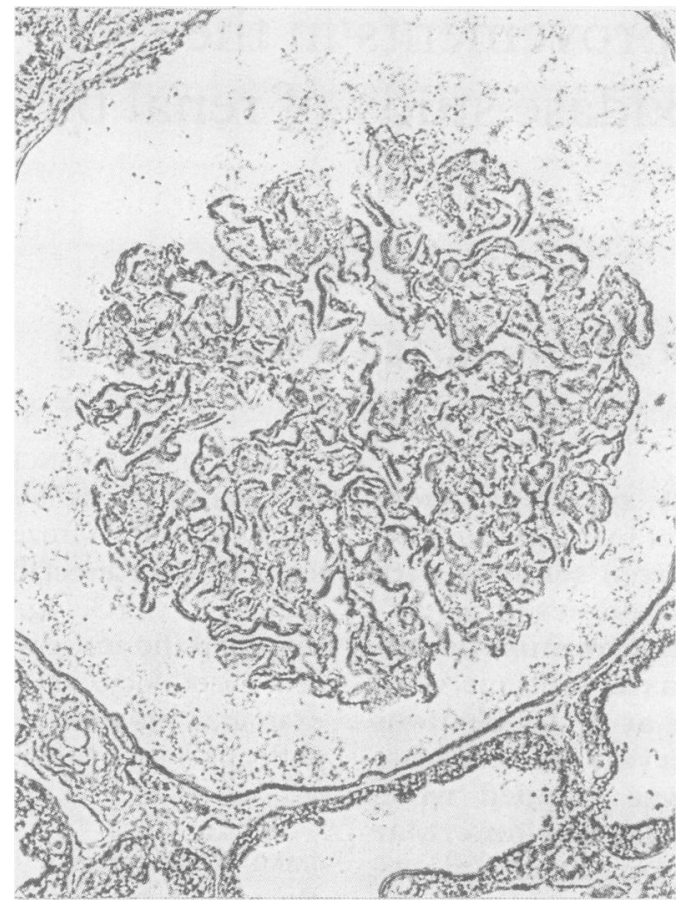

Figure 1 Glomerulus in a renal biopsy specimen before proteolytic digestion, unstained and examined by orthodox microscopy, with the condenser adjusted to give maximum contrast.

biopsy specimen was removed and examined, and so on until digestion was complete. Slides were then transferred to running water for five minutes.

\section{COMPLEMENT COMPONENT C 9}

The indirect immunoperoxidase technique was applied using sheep antiserum to C9 (The Binding Site Limited, Birmingham, England). The optimal titre was found using various dilutions on sections of a case of $\operatorname{IgA}$ nephropathy that contained mesangial deposits of $\mathrm{C} 3$ by both immunofluorescence and immunoperoxidase.

\section{Results}

The time required for optimal digestion of sections with protease varied from five to 60 minutes. There was insufficient correlation between digestion time and treatment of biopsy specimens before sectioning, such as length or type of fixation, to allow standardised digestion times to be preset with confidence. Optimal digestion produced no staining of plasma in capillary loops but permitted intense staining of immune deposits (fig 3 ).

COMPARISON BETWEEN IMMUNOPEROXIDASE AND IMMUNOFLUORESCENCE

Results are shown in table 1. There was

Table 1 Comparison between immunofluorescence (IF) and immunoperoxidase (IP) techniques in analysis of 65 renal biopsy specimens

\begin{tabular}{lllll}
\hline & $I F+$ & $I F-$ & $I F+$ & $I F-$ \\
& $I P+$ & $I P-$ & $I P-$ & $I P+$ \\
\hline Individual immunoproteins: & & & & \\
$\quad$ IgG & 18 & 39 & 4 & 4 \\
IgA & 15 & 48 & 1 & 1 \\
IgM & 21 & 39 & 3 & 2 \\
C3 & 11 & 38 & 16 & 0 \\
Individual biopsy specimens (combined immunoproteins) & 11 & 23 & 24 & 7 \\
\hline
\end{tabular}

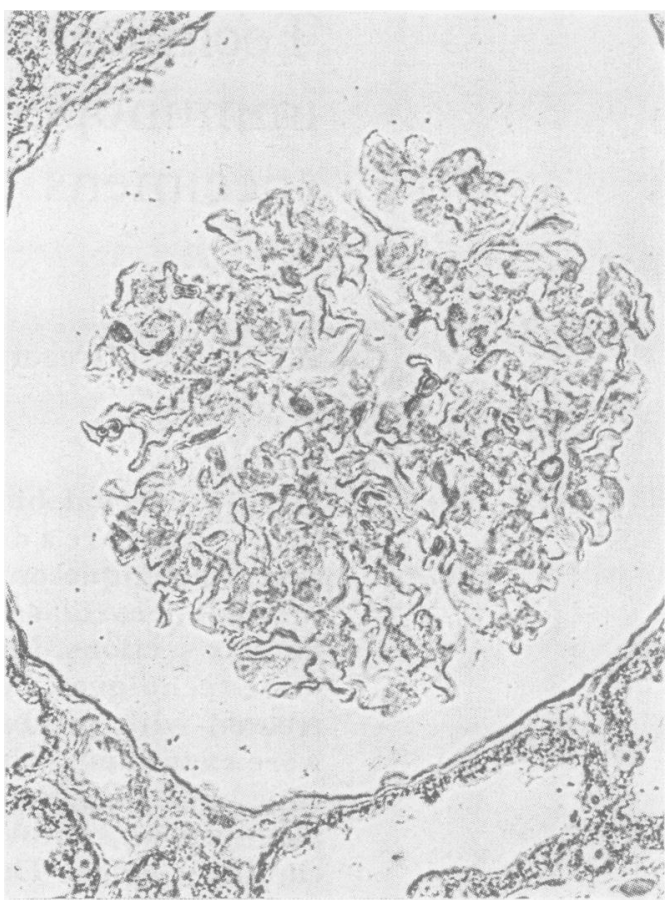

Figure 2 The same glomerulus after optimal digestion. Glomerular basement membranes appear sharp. Plasma has been removed from capillary loops and debris from Bowman's space.

agreement in overall staining between immunoperoxidase and immunofluorescence in 34 biopsy specimens and disagreement in 31 . Discordant results were obtained in 16 biopsy specimens in which C3 was detected by immunofluorescence alone. The other discordant results $(n=15)$ included five with focal segmental lesions containing immunoglobulins not present in every glomerulus, four cases of IgA nephropathy in which IgG or $\operatorname{IgM}$ were discordant, and six cases of other types of glomerulonephritis in which IgG or IgA were

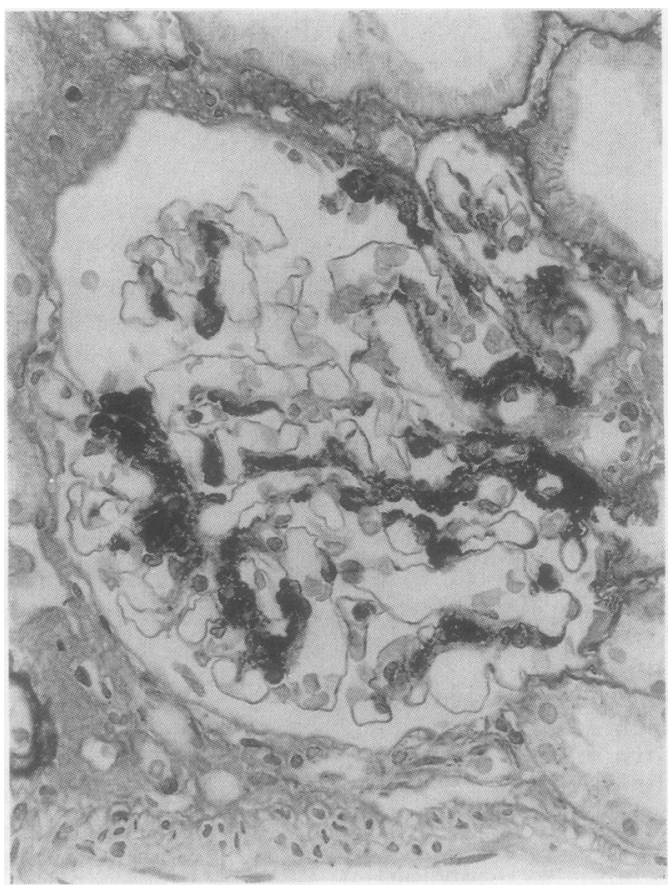

Figure 3 Glomerulus in a renal biopsy specimen stained by the immunoperoxidase technique for $\operatorname{Ig} A$. There is intense mesangial deposition, indicating $\operatorname{Ig} A$ nephropathy. 
Figure 4 Glomerulus in a renal biopsy specimen stained by the immunoperoxidase technique for C9. There is regular, granular deposition of $C 9$ on the external aspect of glomerular capillary loops, indicating membranous nephropathy. $C 9$ is also detected in an arteriole.

Table 2 Comparison between staining for $C 3$ and $C 9$ in 34 renal biopsy specimens

\begin{tabular}{lllll}
\hline & \multicolumn{4}{l}{ Immunoperoxidase findings } \\
\cline { 2 - 5 } Biopsy specimens & $C 3+$ & $C 3-$ & $C 3+$ & $C 3-$ \\
\hline Examined by both techniques $(\mathrm{n}=17)$ & $C 9+$ & $C 9-$ & $C 9-$ & $C 9+$ \\
Immunofluorescence findings: & & & & \\
$\quad \mathrm{C3}+(\mathrm{n}=15)$ & 7 & 0 & 0 & 8 \\
$\mathrm{C3}-(\mathrm{n}=2)$ & 0 & 2 & 0 & 0 \\
Examined by immunoperoxidase alone $(\mathrm{n}=17)$ & 5 & 6 & 0 & 6 \\
\hline
\end{tabular}

discordant. In none of the 65 specimens was a diagnosis given by immunofluorescence but missed by immunoperoxidase; nor did the discrenancies in immunoglobulins affect the
4). In most biopsy specimens arterial and arteriolar walls stained and there was patchy staining of tubular basement membranes. Globally sclerosed glomeruli usually stained and Bowman's capsule occasionally stained.

Results are shown in table 2 . Immunoperoxidase showed $\mathrm{C} 9$ in all 15 biopsy specimens that contained C3 on immunofluorescence, and in six biopsy specimens that lacked $\mathrm{C} 3$ in the cases examined by immunoperoxidase alone.

\section{Discussion}

The method of observation of the digestion of sections required practice but once the optimal stage was recognised the method saved time by eliminating the need to repeat cases that had been underdigested or overdigested. ${ }^{1}$ The immunoperoxidase method gave results with immunoglobulins which were consistent, reliable, and equivalent to those of immunofluorescence, with all the advantages of using fixed material. ${ }^{1}$

C3 was frequently missed by immunoperoxidase. $\mathrm{C} 9$ has been found at the same sites as C3 on frozen sections of renal biopsy specimens, ${ }^{4-6}$ and we have now shown the usefulness of detecting $\mathrm{C} 9$ on immunoperoxidase sections. Other studies also found C9 outside glomeruli in the sites where we found it, ${ }^{56}$ but glomerular deposition was so obvious that interpretation was not difficult.

1 Maclver AG, Mepham BL. Immunoperoxidase techniques in human renal biopsy. Histopathol 1982;6:249-67.

2 Johnson GD. Immunofluorescence techniques. In: Thompson RA, ed. Techniques in clinical immunology. 2nd ed. Oxford: Blackwell Scientific Publications, 1981:106-37.

3 Jones EL, Gregory J. Immunoperoxidase methods. In: Catty D, ed. Antibodies: a practical approach. Vol 2.

4 Biesecker G, Katz S, Koffier D. Renal localization of the membrane attack complex in systemic lupus ery-
thematnsus nenhritic I Fxn Med 1981:154:1779-94. 